\title{
Can Bovine Leukemia Virus Be Related to Human Breast Cancer? A Review of the Evidence
}

Lucia Martinez Cuesta, 1

Pamela Anahi Lendez, 1

Maria Victoria Nieto Farias,

Guillermina Laura Dolcini, 1

Maria Carolina Ceriani, $1 \square$

Phone: 0249154620834

Email: cceriani@gmail.com

${ }^{1}$ Laboratorio de Virología, Facultad de Cs. Veterinarias-UNCPBA, CIVETANCONICET, CICPBA, Campus Universitario, Paraje Arroyo Seco s/n, 7000 Tandil, Argentina

Received: 12 February 2018 / Accepted: 9 May 2018

\section{Abstract}

The incidence of breast cancer is continuously increasing worldwide, as influenced by many factors that act synergistically. In the last decade there was an increasing interest in the possible viral etiology of human breast cancer. Since then, many viruses have been associated with this disease (murine mammary tumor virus, MMTV; Epstein-Barr virus, EBV; and human papillomavirus, HPV). Recently, BLV has been identified in human breast cancers giving rise to the hypothesis that it could be one of the causative agents of this condition. BLV is a retrovirus distributed worldwide that affects cattle, causing lymphosarcoma in a small proportion of infected animals. Because of its similarity with human retroviruses like HTLV and HIV, BLV was assumed to also be involved in tumor emergence. Based on this assumption, studies were focused on the possible role of BLV in human breast cancer development. We present a compilation of the 
current knowledge on the subject and some prospective analysis that is required to fully end this controversy.

\section{Keywords}

Bovine leukemia virus

Breast cancer

Human

Zoonosis

\section{Introduction}

Breast cancer is a multifactorial disease that affects millions of women worldwide [1] and its incidence is increasing continuously [2]. Globally, it is the most frequent cancer in females, and every year, 1.7 million new cases are diagnosed [3]. Breast cancer is about 100 times less common among men than among women, where men have a lifetime risk of about 1 in 1000 [4]. In developed countries, breast cancer ranks in fourth place as the cause of death in women, while in developing countries it falls to sixth place. [5]. There are several risk factors associated with this pathology such as exposure to high levels of estrogen (through the use of hormonal contraceptives or hormone replacement therapy), radiation, age, race, number of births, and obesity after menopause, among others [6, 7]. Moreover, it is known that mutations on several genes such as BRCA1/2, TP53, PTEN, STK11, CDH1, confer a higher risk for developing breast cancer [8].

\section{$\mathrm{AQ1}$}

In particular, specific inherited mutations in BCRA1 and 2 genes notably increase the risk of female breast and ovarian cancer. In a recent large study, it was shown that $72 \%$ of women who inherit a harmful BRCA1 mutation and about $69 \%$ of women who inherit a harmful $B R C A 2$ mutation will develop breast cancer by the age of 80 [9].

Many viruses have been associated with human cancers and it is believed that approximately $12 \%$ of cancers have a viral etiology [10]. In most cases, viral infection is necessary, but not enough for the development of the tumor. These viruses usually have a wide distribution. However, they only cause cancer in a small percentage of individuals, suggesting that other factors influence tumorigenesis [11]. 
The possible viral etiology of breast cancer had its onset with the discovery of MMTV as the causative agent of murine breast cancer. Since then, many viruses such as EBV, HPV and MMTV-like sequences have been identified in human mammary tumors $[12,13]$. A study in Colombia identified BLV $51 \mathrm{kD}$ surface glycoprotein (gp51) of the viral envelope in histological samples of human breast cancer by immunohistochemistry [14]. In 2014, researchers from California detected, for the first time, transcripts of the BLV gene tax in human breast cancer using in situ PCR in slides obtained from formalin-fixed, paraffin-embedded tissues (FFPE) [15]. The information presented above raises the hypothesis that BLV could play a role in the development and/or progression of breast cancer. The aim of this work is to review the knowledge about the virus and to analyze the latest findings on the possible relationship of BLV and human breast cancer.

\section{The Virus}

BLV belongs to the Retroviridae family and is the causative agent of bovine enzootic leukemia. This $\delta$-retrovirus is closely related to the human T-lymphotropic virus type 1 (HTLV-1). The virus' particles range from 60 to $125 \mathrm{~nm}$, with an envelope, an icosahedral capsid and diploid positive sense RNA genome. All the genetic information for completing the viral cycle is flanked by two identical long terminal repeats (LTRs). Apart from the structural proteins, the virus encodes regulatory proteins such as Tax, Rex, G4 and G3, and also expresses regulatory microRNAs [16].

The main target cell in cattle is the $\mathrm{IgM}^{+} / \mathrm{CD}^{+} \mathrm{B}$-lymphocyte. Nevertheless, it can also infect other cells such as monocytes, macrophages and T-lymphocytes [17]. Most of the infected animals are asymptomatic and do not suffer alterations in lymphocyte count. These animals can only be identified as infected by detecting the presence of anti-BLV antibodies or by measuring the proviral load in blood samples [18]. Approximately $30 \%$ of the infected animals develop persistent lymphocytosis (PL), a condition characterized by a benign clonal expansion of B-lymphocytes. Animals with PL usually remain stable for long periods or even for their entire life. However, by mechanisms still unknown, less than $10 \%$ progress to lymphosarcoma, a terminal phase of the disease [19].

Contact with infected cells is the only means of transmission because free BLV particles are unstable and they are not efficient in the infection process. The main route of transmission between animals is iatrogenic mainly by the use of contaminated needles, transrectal palpation, dehorning and tattooing [20]. 
Vertical transmission occurs in 4 to $18 \%$ of the cases and the risk is higher in animals borne from PL mothers [21]. In an experiment carried out with infected cows, high proviral loads in colostrum correlated with high proviral loads in blood, leading to higher risk of transmission [22]. However, other studies support that maternal antibodies contained in milk and colostrum protect the dam and minimize transmission by this route $[23,24]$.

The viral cycle is well known in bovines. After viral recognition through a membrane receptor, membrane fusion occurs, allowing the liberation of the capsid into the cytoplasm of the host cell. Viral reverse transcriptase synthesizes a double strand DNA using the viral RNA as template, with a short DNA-RNA hybrid step. This molecule of DNA forms a complex with the integrase and other cellular proteins to introduce the viral genome into the nucleus, which finally integrates into the host genome. Once integrated, the provirus codifies for nucleic acid and proteins to assemble new viral particles. These particles are released by exocytosis to produce new infective virions [25]. Due to viral integration into the host genome, each cell division originates a new infected cell.

Insertion of BLV nucleic acid frequently occurs in intronic regions. However, there is no specific site for insertion. It has been described that insertion in these low transcriptional regions helps to maintain virus latency, allowing for low expression levels that are enough to stimulate the host immune response [26]. It is believed that cells that are actively expressing virus are detected by the immune system. On the other hand, cells that carry provirus in transcriptionally-inactive sites are not recognized by the immune response. Thus, the infected cells accumulate causing lymphocytosis or proliferation of B-cells, a condition that characterizes this disease [27].

\section{Could BLV Infect Humans?}

After the discovery of BLV, antibodies against this virus were not detected in humans. Therefore, the idea of a possible zoonosis was discarded. However, the development of new and more sensitive diagnostic methods allowed the detection of antibodies against viral proteins in human sera [28]. These findings were the starting point in the debate about the possibility of BLV infecting humans. The presence of these antibodies only implies that humans are exposed to BLV but does not confirm an infection. 
For an infection to occur there must be a receptor that allows virus to enter the cell. Transmembrane proteins that internalize different substances are used as receptors by several members of the Retroviridae family [29]. The closely related virus HTLV-1 uses the glucose transporter GLUT-1 as a cell receptor [30]. In the case of $\mathrm{BLV}$, scientists have not yet identified the receptor. It is believed that the receptor is the $\delta$ subunit of bovine adaptor protein complex 3 (AP-3) [31]. Humans have four types of AP complexes that are widely distributed in the body and have an important role in intracellular transport [32]. The existence of a human homolog of bovine BLV receptor means that there is a chance that BLV can infect this species. In fact, scientists were able to infect human cell lines with the virus in vitro [33].

The exact way by which the virus might be transmitted to humans is still unknown. It was proposed that unpasteurized milk or undercooked meat could be a way in which the virus enters the body. It has been reported that the genetic material corresponding to the viral gene gag is present in fresh milk and raw meat [34]. However, it is well known that pasteurization and cooking inactivate this virus. Nevertheless, it is possible that the virus was transmitted to humans many years ago, when bovine products were uncontrolled [35]. Once the virus was integrated into the host genome, it is likely that transmission from person to person occurred in the same way as its counterpart HTLV (blood, semen and breast milk) [36].

\section{What Do we Know about BLV in Human Cancer?}

The Retroviridare family includes HIV, HTLV-1 and BLV, which share structural and functional homology. Both human viruses, HIV and HTLV, had been classified as a group I carcinogenic virus by the International Agency for Research on Cancer (IARC) [37]. BLV belongs to the same family as these human carcinogenic viruses. Thus, it is possible that BLV could be carcinogenic in humans. This possibility has not yet been studied thoroughly.

Viruses are classified into direct and indirect oncoviruses: while HTLV is a direct oncovirus, HIV is considered an indirect oncovirus. This classification is based on the fact that the genome of direct oncoviruses can be detected in tumor cells, expressing proteins that cause cell transformation and immortalization. On the other hand, indirect oncoviruses contribute to the development of cancer through chronic inflammation and immunosuppression, usually occurring after decades of infection.

There are many similarities between BLV and HTLV-1. Both cause persistent infections, $\mathrm{h}$ infect lymphocytes and are transmitted by direct contact with infected 
cells [19]. Intrauterine transmission is low because infected lymphocytes do not pass efficiently through the placenta. In HTLV-1, breast feeding is the main route of mother-to-child viral transmission, whereas it remains controversial as to whether this is an important route of transmission for BLV, although it is a fact that transmission in uterus from cow to calf is possible [23, 24, 38].

In both cases, tumor development is not related to the integration site in the host genome [39]. The viral protein Tax plays an important role in tumorigenesis, as it stimulates cell survival activating the transcription factor $\mathrm{NF}-\kappa \mathrm{B}$, and also increases the expression of anti-apoptotic proteins (such as Bcl-2) [40] and the expression of TNF- $\alpha$ [41]. However, the effect of Tax is not enough for tumor development; other factors such as mutations in the tumor-suppressor protein p53 [16] might be necessary. Both BLV and HTLV-1 only induce leukemia or lymphoma in a low percentage $(1-5 \%)$ of the infected individuals and this occurs years after initial infection $[18,42]$.

\section{Why Do we Associate BLV with Breast Cancer?}

Due to the discovery of BLV p24 and viral DNA in bovine mammary tissue, scientists considered the possibility that BLV could be found in human in the same tissue [43]. A first attempt to identify BLV in human breast was made by Buehring and collaborators who analyzed 219 FFPE breast tissue samples. The viral gene tax was detected in $44 \%(n=97)$ of samples from human breast cancer analyzed by in situ PCR. In 12 out of 215 samples (6\%), the $24 \mathrm{kD}$ viral protein was detected by immunohistochemistry. These results suggest that, in some way, the virus could be active because p24 is a marker of viral replication [15].

The viral gene env encodes an envelope glycoprotein, gp51, which has a major role in the infection process [18]. In 2006, Cruz and collaborators analyzed 56 samples from human breast cancer by immunohistochemistry for gp51. They detected this protein in $7 \%$ of the samples (4/56) [14]. The presence of gp51 is also indicative of viral replication.

In a retrospective study, 53 human samples of FFPE breast cancer tissue and 53 control samples of the same tissue stored at a hospital in Bogota, Colombia, were analyzed. By conventional PCR, they were able to amplify a fragment of the viral gene gag, which codifies for the viral capsid [44], in 35\% of the breast cancer samples and $45 \%$ of the control tissues [45]. The odds ratio, which indicates the possibility of the disease occurring in people exposed and not exposed to BLV, did not establish an association between BLV and breast cancer. However, they found 
that samples from older patients had a greater likelihood of being positive for BLV sequences, similar to the findings by Buehring [35]. This is an important fact if we consider that people exposed to the virus is supposed to occur through dairy and meat. While growing older, the possibility of accumulating more events of exposure through continuous consumption of these foods could be increased.

A similar analysis, performed in 2015, analyzed viral gene tax expression using in situ PCR. The target gene was amplified in $29 \%$ of the controls, $38 \%$ of the premalignant lesions and $59 \%$ of malignant samples. The odds ratio of the association between the presence of BLV DNA and breast cancer was 3.07, a value similar to that obtained for other risk factors such as hormonal exposure, number of births and lifestyle [35].

Recently, a study conducted in Australia detected the presence of a BLV gene before and after breast cancer development. These results showed that viral DNA could be detected in this tissue many years before the onset of the disease. In 10 cases, it was possible to obtain enough DNA to sequence the viral LTR. The LTR sequences were compared to a reference sequence. After comparison, authors showed that in 6 samples the LTRs had a 100\% homology with the reference sequence and 4 had at least one substitution [46].

On the other hand, other studies show absolutely different results. These studies disagree with the findings that imply an association between BLV and breast cancer. In a thorough study conducted in China in 2016 on 91 breast cancer tissues and 160 blood samples from women with a breast cancer diagnosis, it was not possible to detect BLV transcripts or antibodies against the virus [47]. In analyzing these results, Buehring concluded "we used in situ PCR, which requires sample preparation, cycling times/temperatures, reaction mix composition, and a thermal cycler different from those used for standard solution PCR and real-time PCR. It is impossible from the information given to evaluate whether the authors' in situ PCR techniques were equivalent to what we used" [48]. Furthermore, the commercial ELISA kit they used was specially intended for cattle. These kits usually are standardized and extensively tested for the level of anti-BLV antibodies usually found in BLV-infected cattle, which is much higher than in humans [28, 49].

Another study, performed by Gillet and collaborators, analyzed the complete genome of 51 breast tumors obtained from the NCBI database (dbGaP, NCBI database of genotype and phenotype). In this case, they evaluated the presence of BLV sequences by aligning the retrieved sequences with different BLV strains, using human genomic sequences as the positive control. They used the "very 
sensitive" option of Bowtie2 to maximize likelihood of viral detection, and created "in silico" divergent sequences. They had no positive results, no matter the technology used for analysis [50].

Recently, a case control study of women in Texas supports the results found in previous studies showing an association of BLV DNA with breast cancer. They detected a fragment of the tax gene in $20 \%$ of benign, $34 \%$ of pre-malignant, and $57 \%$ of malignant tissues. Moreover, they assessed HPV presence in these samples. Their conclusion was that there is no association of oncogenic strains of HPV with either breast cancer development or with BLV presence [51].

\section{Will there Be Solid Proof that BLV Is Associated with Breast Cancer?}

The development of cancer is the result of complex interactions between genetic and epigenetic factors. Direct oncogenic viruses contribute to the development or progression of certain cancers favoring the accumulation of mutations or preventing tumor cell removal by blocking the expression of apoptotic genes. In addition to direct mechanisms, chronic viral infections create an inflammatory environment that indirectly contributes to tumor formation. With all this information in mind, it might be possible that BLV plays a role in human breast cancer given that it produces persistent infections and has functional and structural similarities with HTLV-1, a human direct oncovirus $[18,27,52]$.

Detecting the viral DNA in breast cancer tissue is only a beginning link to any association between BLV and this type of cancer. The human genome typically contains thousands of endogenous retroviruses, many of which are derived from retroviruses that circulated millions of years ago. This is why in a first instance it should be discarded that the detected BLV sequences come from that source. To establish a causal relationship there must be prospective studies demonstrating that viral infection precedes tumor development. In this case, there is only one prospective study carried out in Australia that should be validated with other research [46]. Another future perspective is to identify the precise route of virus transmission to humans and to determine how many infected individuals develop cancer.

In any case in which a virus is associated with cancer development (such as HTLV1, HPV, EBV or hepatitis C virus), viral presence should be accompanied by other factors inherent to the individual and the environment which stimulates cellular transformation [53]. One example is EBV, whose prevalence worldwide is around 
$90 \%$. However, the incidence of Burkitt's lymphoma, which is associated with EBV infection, is higher in Africa. This could be due to environmental or genetic factors that make people in Africa more susceptible to the development of this cancer [54]. It would be interesting to evaluate the contribution of each of these factors to the development or progression of breast cancer in association with BLV. This may explain why not all people exposed to this virus develop cancer.

In 1965, Sir Austin Bradford Hill described criteria to evaluate causal association between environmental and occupational exposures and the development of certain diseases. These nine criteria are also used to evaluate a virus' oncogenic potential. Due to the complexity of cancer (where viral, host and environmental factors are involved) it is not necessary to fulfill all the criteria in order to associate a virus with a cancer.

Hill's criteria includes: consistence of the observation; strength of the association; specificity; temporality; biological gradient (if the incidence of the disease increases with the exposition), biological feasibility; coherence of the association; experimental evidence and analogy of the exposition with other well-known cause of the disease [55].

In the case of BLV, several criteria, but not all, are met, such as strength of association and consistency: some parts of the virus have been detected in different populations and by different methodologies [14, 15, 35, 45, 46]. The temporality was proven by Buehring and her Australian counterpart: they detected viral sequence in mammary tissue many years before tumor development. Moreover, in some cases they could not detect BLV sequence in the first sample taken from the patient but were able to identify viral transcripts in tumor samples from the same individual after several years [46]. These results might support the idea of a causal relationship between BLV and the further development of breast cancer.

Lastly, following analogy criteria, BLV and HTLV structural and functional similarity can let scientists consider the possibility that BLV could affect humans in some way. BLV and HTLV are both members of the Deltaretrovirus genus, characterized by the presence of a unique sequence called $p X$ region located between the env gene and the 3'LTR. This sequence encodes regulatory proteins, like Tax and Rex that are not present in other retroviruses [16]. These similarities support the hypothesis that continuous contact between BLV and humans could lead to a virus adaptation to this new host. 
Many studies in this field have been performed by different groups, with dissimilar conclusions. The fact that some researchers could not find the virus in breast cancer patients could be related to the methodology used and the population in which the study was conducted.

Zhang and collaborators developed their research in different regions of China. It is known that milk consumption in the Chinese population is four times lower than in Argentina and almost 10 times lower than in industrialized countries like Australia, New Zeland, or the United States [56]. There is a correlation between bovine milk and meat consumption and breast cancer. Countries with high consumption of these products have a higher prevalence of breast cancer than countries like China or India that consume fewer products derived from cattle. Moreover, women with lactose intolerance have a lower prevalence of breast cancer than other women and other family members, reinforcing the importance of considering milk consumption habits when analyzing the population in a study [57].

Many facts suggest that it is unlikely to detect retroviral sequences by next generation sequencing techniques. Despite the enormous amount of data that can be obtained through high-throughput shotgun sequencing, viral DNA in a typical clinical sample usually represents a very low proportion of the host genome [58]. Another possibility could be that the samples analyzed had a low number of infected cells, or that, despite being infected, the level of integrated retrovirus was too low. More research in human database needs to be done. Due to the quantitative disproportion between viral and host genomic material, previous enrichment of the target by in-solution or solid-surface hybridization could be a good approach [59]. In addition, it cannot be assumed that viruses associated with human breast cancer, like MMTV, HPV or BLV, exert their action by continuously expressing their proteins [58].

On the other hand, BLV sequences were detected in tissue control samples that came from reductive surgeries. The authors consider that these tissues could be in early stages of a neoplasia that might be evident clinically many years later [35, 46]. This is consistent with viral action in cattle, where it maintains a low transcriptional rate to escape from the immune system and provokes lymphoma many years after initial infection [18]. As previously described, the BLV oncogenic effect is not related with the expression of oncoproteins or its place of insertion in the host genome. The viral protein Tax has a major role in oncogenesis, affecting some DNA damage repair mechanisms that contribute to an accumulation of 
mutations [16]. This could explain why, both in cattle and human, tumors appear many years after initial contact with the virus.

\section{Conclusions}

Regarding the arguments presented in this review, there is not a consensus about the possible relationship between BLV and human breast cancer. To end these controversies, it is essential to isolate an infective viral particle from a breast tumor, from human breast milk, or from meat for human consumption.

Identification of a link between BLV and breast cancer pathogenesis would improve preventive strategies were a link proven [60]. Confirming that BLV is related to human breast cancer would be of great importance as it is a worldwide-distributed virus in cattle and there are several instances in which humans can be exposed. More research has to be done to confirm if BLV is associated with breast cancer in humans. If, indeed, there is a relation between BLV and cancer, then it will be peremptory for governments to design eradication plans, mostly to reassure the population. If this relation does not exist, then it will be necessary to understand why fragments of the BLV genome can be detected in human tissue.

\section{References}

1. Ferlay J, Shin HR, Bray F, Forman D, Mathers C, Parkin DM. Estimates of worldwide burden of cancer in 2008: GLOBOCAN 2008. Int J Cancer. 2010;127(12):2893-917.

2. Tao Z, Shi A, Lu C, Song T, Zhang Z, Zhao J. Breast Cancer: epidemiology and etiology. Cell Biochem Biophys. 2015;72(2):333-8.

3. Ferlay J, Soerjomataram I, Dikshit R, Eser S, Mathers C, Rebelo M, et al. Cancer incidence and mortality worldwide: sources, methods and major patterns in GLOBOCAN 2012. Int J Cancer. 2015;136(5):E359-86.

4. American Cancer Society. Cancer facts \& figures 2017. Atlanta: American Cancer Society; 2017.

5. Global Burden of Disease Cancer Collaboration, Fitzmaurice C, Dicker D, Pain A, et al. The global burden of cancer 2013. JAMA Oncol. 2015;1(4):50527. 
6. Kamińska M, Ciszewski T, Łopacka-Szatan K, Miotła P, Starosławska E. Breast cancer risk factors. Prz Menopauzalny. 2015;14(3):196-202.

7. Torre LA, Bray F, Siegel RL, Ferlay J, Lortet-Tieulent J, Jemal A. Global cancer statistics. CA Cancer J Clin. 2015;65(2):87-108.

8. Campeau PM, Foulkes WD, Tischkowitz MD. Hereditary breast cancer: new genetic developments, new therapeutic avenues. Hum Genet. 2008;124(1):3142.

9. Kuchenbaecker KB, Hopper JL, Barnes DR, Phillips KA, Mooij TM, RoosBlom MJ, et al. Risks of breast, ovarian, and contralateral breast cancer for BRCA1 and BRCA2 mutation carriers. JAMA. 2017;317(23):2402-16.

10. Parkin DM. The global health burden of infection-associated cancers in the year 2002. Int J Cancer. 2006;118:3030-44.

11. Sarid R, Gao SJ. Viruses and human cancer: from detection to causality. Cancer Lett. 2011;305(2):218-27.

12. Di Lonardo A, Venuti A, Marcante ML. Human papillomavirus in breast cancer. Breast Cancer Res Treat. 1992;21(2):95-100.

13. Wang Y, Holland JF, Bleiweiss IJ, Melana S, Liu X, Pelisson I, et al. Detection of mammary tumor virus env gene-like sequences in human breast cancer. Cancer Res. 1995;55(22):5173-9.

14. Ochoa Cruz A, Uribe A, Gutiérrez M. Estudio del potencial zoonótico del virus de la leucosis bovina y su presencia en casos de cáncer de seno. Univ Sci. 2006;11(2):31-40.

15. Buehring GC, Shen HM, Jensen HM, Choi KY, Sun D, Nuovo G. Bovine leukemia virus DNA in human breast tissue. Emerg Infect Dis. 2014;20(5):77282.

16. Aida Y, Murakami H, Takahashi M, Takeshima SN. Mechanisms of pathogenesis induced by bovine leukemia virus as a model for human T-cell leukemia virus. Front Microbiol. 2013;4:328. 
17. Panei CJ, Takeshima S, Omori T, Nunoya T, Davis WC, Ishizaki H, et al. Estimation of bovine leukemia virus (BLV) proviral load harbored by lymphocyte subpopulations in BLV-infected cattle at the subclinical stage of enzootic bovine leucosis using BLV-CoCoMo-qPCR. BMC Vet Res. 2013;9:95.

18. Gillet N, Florins A, Boxus M, Burteau C, Nigro A, Vandermeers F, et al. Mechanisms of leukemogenesis induced by bovine leukemia virus: prospects for novel anti-retroviral therapies in human. Retrovirology. 2007;4:18.

19. Rodríguez SM, Florins A, Gillet N, de Brogniez A, Sánchez-Alcaraz MT, Boxus M, et al. Preventive and therapeutic strategies for bovine leukemia virus: lessons for HTLV. Viruses. 2011;3:1210-48.

20. Hopkins SG, Di Giacomo RF. Natural transmission of bovine leukemia virus in dairy and beef cattle. Vet Clin North Am Food Anim Pract. 1997;13(1):10728.

21. Lassauzet ML, Thurmond MC, Johnson WO, Stevens F, Picanso JP. Factors associated with transmission of bovine leukemia virus by contact in cows on a California dairy. Am J Epidemiol. 1991;133(2):164-76.

22. Gutiérrez G, Lomonaco M, Alvarez I, Fernandez F, Trono K. Characterization of colostrum from dams of BLV endemic dairy herds. Vet Microbiol. 2015;177(3-4):366-9.

23. Van Der Maaten MJ, Miller JM, Schmerr MJF. Effect of colostral antibody on bovine leukemia virus infection of neonatal calves. Am J Vet Res. 1981;42:1498-500.

24. Van der Maaten MJ, Miller JM, Schmerr MJ. In utero transmission of bovine leukemia virus. Am J Vet Res. 1981 Jun;42(6):1052-4.

25. Lewinski MK, Bushman FD. Retroviral DNA integration--mechanism and consequences. Adv Genet. 2005;55:147-81.

26. Murakami H, Yamada T, Suzuki M, Nakahara Y, Suzuki K, Sentsui H. Bovine leukemia virus integration site selection in cattle that develop leukemia. Virus Res. 2011;156(1-2):107-12. 
27. Florins A, Gillet N, Asquith B, Boxus M, Burteau C, Twizere JC, et al. Cell dynamics and immune response to BLV infection: a unifying model. Front Biosci. 2007;12:1520-31.

28. Buehring GC, Philpott SM, Choi KY. Humans have antibodies reactive with Bovine leukemia virus. AIDS Res Hum Retrovir. 2003;19(12):1105-13.

29. Sommerfelt MA. Retrovirus receptors. J Gen Virol. 1999;80:3049-64.

30. Manel N, Kim FJ, Kinet S, Taylor N, Sitbon M, Battini JL. The ubiquitous glucose transporter GLUT-1 is a receptor for HTLV. Cell. 2003;115(4):449-59.

31. Suzuki T, Matsubara Y, Kitani H, Iked H. Evaluation of the d subunit of bovine adaptor protein complex 3 as a receptor for bovine leukaemia virus. J Gen Virol. 2003;84:1309-16.

32. Robinson MS, Bonifacino JS. Adaptor-related proteins. Curr Opin Cell Biol. 2001;4:444-53.

33. Graves DC, Ferrer JF. In vitro transmission and propagation of the bovine leukemia virus in monolayer cell cultures. Cancer Res. 1976;36:4152-9.

34. Olaya-Galán NN, Corredor-Figueroa AP, Guzmán-Garzón TC, RíosHernandez KS, Salas-Cárdenas SP, Patarroyo MA, et al. Bovine leukaemia virus DNA in fresh milk and raw beef for human consumption. Epidemiol Infect. 2017; 145:3125-30.

35. Buehring GC, Shen HM, Jensen HM, Jin DL, Hudes M, Block G. Exposure to bovine leukemia virus is associated with breast cancer: a case-control study. PLoS One. 2015;10(9):e0134304.

36. Gross C, Thoma-Kress AK. Molecular mechanisms of HTLV-1: cell-to-cell transmission. Viruses. 2016;8(3):74.

37. IARC Working Group on the Evaluation of Carcinogenic Risks to Humans: Human papillomaviruses. IARC monographs on the evaluation of carcinogenic risks to humans/World Health Organization, International Agency for Research on Cancer . 2007, 90:1-636. 
38. Carneiro-Proietti ABF, Amaranto-Damasio MS, Leal-Horiguchi CF, Bastos RHC, Seabra-Freitas G, Borowiak DR, et al. Mother-to-child transmission of human T-cell lymphotropic viruses-1/2: what we know, and what are the gaps in understanding and preventing this route of infection. J Pediatric Infect Dis Soc. 2014;3(Suppl 1):S24-9.

39. Miyasaka T, Oguma K, Sentsui H. Distribution and characteristics of bovine leukemia virus integration sites in the host genome at three different clinical stages of infection. Arch Virol. 2015;160:39-46.

40. Takahashi M, Tajima S, Okada K, Davis WC, Aida Y. Involvement of bovine leukemia virus in induction and inhibition of apoptosis. Microbes Infect. 2004;7:19-28.

41. Twizere JC, Kruys V, Lefèbvre L, Vanderplasschen A, Collete D, Debacq C, et al. Interaction of retroviral tax oncoproteins with tristetraprolin and regulation of tumor necrosis factor-alpha expression. J Natl Cancer Inst. 2003;95(24):1846-59.

42. Ahmadi Ghezeldasht S, Shirdel A, Assarehzadegan MA, Hassannia T, Rahimi H, Miri R, et al. Lymphotropic virus type I (HTLV-I) oncogenesis: molecular aspects of virus and host interactions in pathogenesis of adult $\mathrm{T}$ cell leukemia/lymphoma (ATL). Iran J Basic Med Sci. 2013;16:179-95.

43. Buehring GC, Kramme PM, Schultz RD. Evidence for bovine leukemia virus in the mammary epithelial cells of infected cows. Lab Investig. 1994;71:359-65.

44. Yoshinaka YK. Bovine leukemia virus protease: pirufication, chemical analysis, and in vitro processing of gag precursos polyproteins. J Virol. 1986;57:826-32.

45. Mesa G, Ulloa JC, Uribe AM, Gutierrez MF. Bovine leukemia virus gene segment detected in human breast tissue. Open J Med Microbiol 2013; 3: (1)8490.

46. Buehring GC, Shen H, Schwartz DA, Lawson JS. Bovine leukemia virus linked to breast cancer in Australian women and identified before breast cancer development. PLoS One. 2017;12(6):e0179367. 
47. Zhang R, Jiang J, Sun W, Zhang J, Huang K, Gu X, et al. Lack of association between bovine leukemia virus and breast cancer in Chinese patients. Breast Cancer Res. 2016;18:101.

48. Buehring GC. Response to "lack of association between bovine leukemia virus and breast cancer in Chinese patients". Breast Cancer Res. 2017;19:24.

49. Choi KY, Liu RB, Buehring GC. Relative sensitivity and specificity of agar gel immunodiffusion, enzyme immunosorbent assay, and immunoblotting for detection of anti-bovine leukemia virus antibodies in cattle. J Virol Methods. 2002;104:33-9.

50. Gillet N, Willems L. Whole genome sequencing of 51 breast cancers reveals that tumors are devoid of bovine leukemia virus DNA. Retrovirology. 2016;13:75.

51. Baltzell KA, Shen HM, Krishnamurty S, Sison JD, Nuovo GJ, Buehring GC. Bovine leukemia virus linked to breast cancer but not coinfection with human papillomavirus: case-control study of women in Texas. Cancer. 2018;124(7):1342-9.

52. Frie MC, Coussensa PM. Bovine leukemia virus: a major silent threat to proper immune responses in cattle. Vet Immunol Immunopathol. 2015;163(34):103-14.

53. Schiller JT, Lowy DR. Virus infection and human cancer: an overview. Recent Results Cancer Res. 2014;193:1-10.

54. Chen CJ, Hsu W, Yang H, Lee M, Chen H, Chien Y, et al. Epidemiology of virus infection and human cancer. Recent Results Cancer Res. 2014;193:11-32.

55. Hill AB. The environment and disease: association or causation? Proc R Soc Med. 1965;58:295-300.

56. He Y, Yang X, Xia J, Zhao L, Yang Y. Consumption of meat and dairy products in China: a review. Proc Nutr Soc. 2016;75:385-91.

57. Lawson JS, Salmons B, Glenn WK. Oncogenic viruses and breast cancer: mouse mammary tumor virus (MMTV), bovine leukemia virus (BLV), human 
papilloma virus (HPV), and Epstein - Barr virus (EBV). Front Oncol. 2018;8:1.

58. Lawson JS, Salmons B, Gunzburg WH. Commentary regarding Gannon et al. "viral infections and breast cancer e A current perspective". Cancer Lett. 2018;424:117e118.

59. Vinner L, Mourier T, Friis-Nielsen J, Gniadecki R, Dybkaer K, Rosenberg J, et al. Investigation of human cancers for retrovirus by low-stringency target enrichment and high-throughput sequencing. Sci Rep. 2015;5:13201.

60. Read SA, Douglas MW. Virus induced inflammation and cancer development. Cancer Lett. 2014;345(2):174-81. 